\title{
An Overview of Flux Pumps for HTS Coils
}

\author{
T. A. Coombs, Senior Member, IEEE, J. Geng, L. Fu, and K. Matsuda
}

\begin{abstract}
High-Tc Superconducting (HTS) flux pumps are capable of injecting flux into closed HTS magnets without electrical contact. It is becoming a promising alternative of current source in powering HTS coils. This paper reviews the recent progress in flux pumps for HTS coil magnets. Different types of HTS flux pumps are introduced. The physics of these flux pumps are explained and comparisons are made.
\end{abstract}

Index Terms-Coated conductor coils, flux pump, HTS.

\section{INTRODUCTION}

$\mathrm{T}$ HANKS to the progress in manufacturing long length Coated Conductors $(\mathrm{CC}), \mathrm{CC}$ coils are becoming more and more popular for magnets. CC coils have much better mechanical properties compared with HTS bulks [1] and have satisfactory current density. $\mathrm{CC}$ coils are also more flexible than tape stacks. These merits make CC coils ideal for high field magnet uses, like in MRI/NMR insert coil [2][3]. In these applications, it is desirable to operate $\mathrm{CC}$ coil magnets in persistent current mode [4], in which case thick current lead and associated heat losses can be avoided. However, flux leakage is inevitable in closed CC coils. This is because although HTS joints with extremely low resistance have been developed [5], flux creep [6] in CC coils is non-negligible, especially when the transport current is close to critical current and the operating temperature is high. Furthermore, when $\mathrm{CC}$ coils are under external AC field, loss is induced which makes the direct current decay much more evident [7][8]. In recent years, HTS flux pumps have been developed to compensate the current decay in $\mathrm{CC}$ coils, which makes it possible for $\mathrm{CC}$ coils to work in persistent current mode.

This paper reviews the recent progress in HTS flux pumps for CC coils. Three main categories of HTS flux pumpstravelling wave flux pumps and transformer-rectifier and variable resistance rectifier flux pumps-are introduced in Chapters II-IV , the general physics of these flux pumps are explained in Chapter V, and the comparisons and applications of these flux pumps are concluded in Chapter VI.

\section{Travelling Wave Flux PuMPS}

\section{A. Travelling magnetic wave flux pumps in general}

Travelling magnetic wave flux pumps have been the most popular flux pumps in recent years. First proposed by Coombs [9][10] The term travelling magnetic wave describes a magnetic

\footnotetext{
Manuscript received on September 6th, 2016.

The authors are with the Electrical Engineering Division, Department of Engineering, University of Cambridge, Cambridge CB3 0FA, U.K. (e-mail: tac1000@cam.ac.uk,jg717@cam.ac.uk,,lf359@cam.ac.uk).
}

field varying with time and in space, in which the waveform is moving relative to the superconductor and which do not rely on switches or a normal spot. This was important because all preceding methods required either a moving normal spot or superconducting switches and this has restricted their use. Thus for example in [12][13][14], Chung proposed a linear electromagnet flux pump for HTS magnets, yet this relied on inducing a moving normal spot in a Niobium sheet. Nakamura [15] proposed a similar arrangement but pointed out that $\mathrm{MgB} 2$ could be used rather than Niobium but their method still required a normal spot. Similarly Bai [16][17][18] achieved flux pumping using a $\mathrm{BSCCO}$ sheet and $\mathrm{MgB}_{2}$ sheet but in [17] they attribute the operation to the motion of a normal spot.

A travelling wave and the equivalent circuit for its operation are shown in Fig. 1.

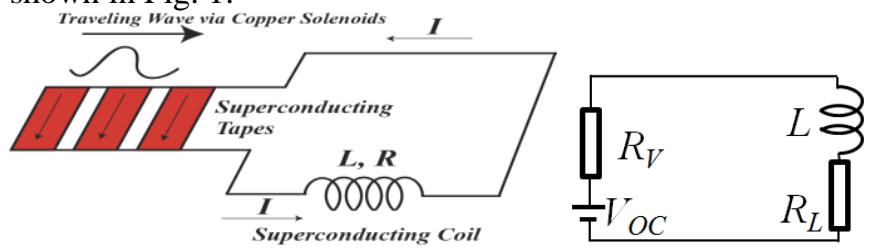

(a) Schematic drawing

(b) equivalent circuit [9]

Fig. 1. Description of travelling wave flux pumps.

$\mathrm{H}(t, x)=\vec{A} \sin 2 \pi(f t-x / \lambda)$ describes a sinusoidal magnetic wave which has a peak value of $A$, a frequency $f$, and wavelength $\lambda$ and is travelling in the $x$ direction. A schematic drawing of travelling wave flux pumps is shown in Fig. 1(a). Typically flux pumps utilize a section of superconductor, the superconductor could be a bulk or more usefully a sheet or sheets (YBCO coated conductors for example) in which the superconducting circuit includes an HTS load coil. A travelling magnetic wave is applied normal to the superconductor, and it repeatedly travels across the superconductor. The waveform could be linear or cylindrical, depending on the geometry of the superconductor. Magnetic flux gradually accumulates in the superconducting circuit. This occurs because a net voltage is induced in the superconducting circuit, as shown in Fig. 1(b) [11].The travelling wave can be generated: thermally, optically; by linearly arranged coils switched in sequence; switched concentric coils[19] or by translating permanent magnets (usually attached to a rotating wheel). This review will concentrate on the linearly arranged coils and the rotating magnet methods.

\section{B. Linear electro-magnets based travelling wave flux pump (LTWFP)}

The travelling magnetic wave in this kind of flux pump is generated by linear electro-magnets [15] [16] [17] [18] [20] [21] [22] An example is shown in Fig. 2, pairs of poles above and below the superconductor are activated in sequence, generating the desired travelling wave in the gap where the superconductor is sited. 


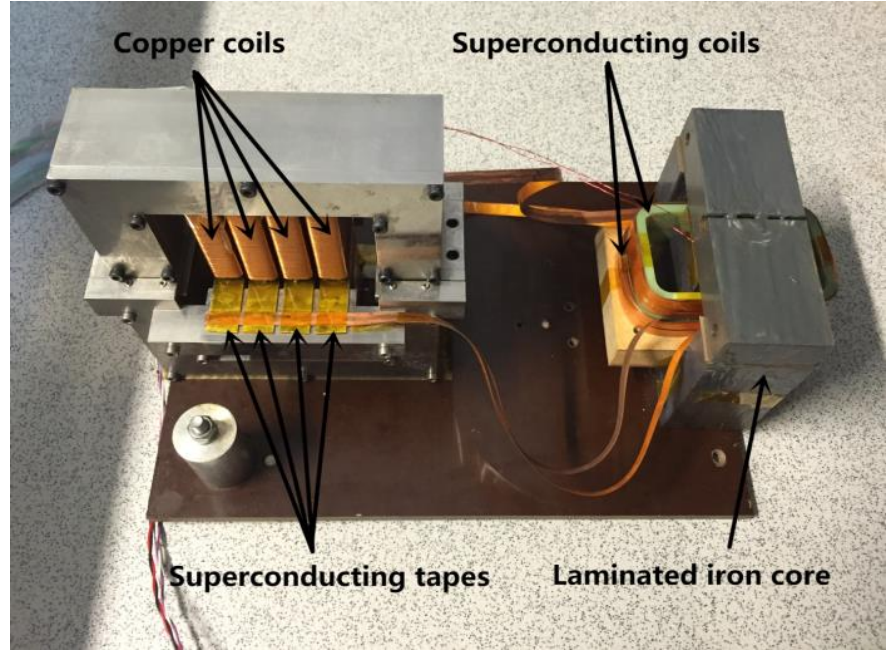

Fig 2. Travelling wave Flux pump

Using this arrangement Lin and co-workers [20][21][22]achieved flux pumping using YBCO coated conductors, and the performance of the flux pump under different field magnitudes, frequencies, and wave profiles was investigated. This system, which used multiple parallel conductors, was the first linear electro-magnetic system in which a normal spot was clearly not the origin of the pumping.

\section{Rotating magnets based travelling wave flux pump (RTWFP)}

RTWFP was firstly proposed by Hoffmann and co-workers [11][23][24]]. Unlike linear electro-magnets based flux pump, the travelling magnetic field in this kind of flux pumps is generated by rotating permanent magnets. As shown in Fig. 3, several permanent magnets are uniformly mounted on a round disk, with the same field polarity facing outward. The disk is driven by a motor through a shaft in the middle of the disk.

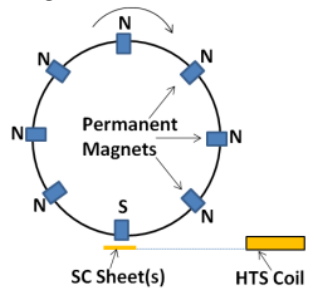

Fig. 3. Schematic drawing rotating permanent magnets based flux pump.

When the disk rotates, each magnet will pass over a superconducting sheet. The sheet thus experiences a travelling magnetic wave. The equivalent circuit of a rotating permanent magnets based flux pump has an open circuit voltage in series with an effective resistance [11][25] The effective resistance observed is much larger than the joint resistances [11][26]. Jiang and co-workers [26] proposed that dynamic resistance contributes most to the effective resistance and is the limiting factor of saturation current. Lee and co-workers [27] reported that the sheet width strongly influences the saturation current. The magnitude of the voltage observed is proportional to rotating frequency, and the polarity is related to rotating direction and the polarity of the magnets [11][26][28][29]. The open circuit voltage and saturation current decrease with the field magnitude drop [28].

\section{Mechanism}

The origin of the nett open circuit voltage of travelling wave flux pumps has been mysterious for years. The key fact is that, unlike low $T_{\mathrm{c}}$ superconductors, high $T_{\mathrm{c}}$ superconductors have a high $H_{\mathrm{c} 2}$, much higher than that provided by either the coils or the permanent magnets (for which it is impossible to generate a normal spot in HTS sheets). How flux can accumulate without generating normal spots is essential to understand these flux pumps. In Section V, this will be discussed in detail.

\section{SWITCHED TRANSFORMER-RECTIFIER PUMPS}

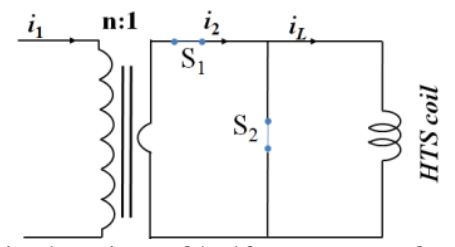

Fig. 4. Schematic drawing of half wave transformer-rectifier flux pumps.

Transformer rectifier flux pumps are common for low $T_{\mathrm{c}}$ superconductors, Klundert et al [30][31] present a variety of types. These type of flux pumps use a transformer and superconducting switches. A half wave rectifier is shown in Fig. 4. The transformer induces high ac current $i_{2}$ in its secondary winding. When the instantaneous value of $i_{2}$ is higher than the load current $i_{\mathrm{L}}$, the switch $\mathrm{S}_{2}$ is opened, and the HTS coil is charged. Then $S_{2}$ is closed to isolate the coil from the flux pump, and $S_{1}$ is opened. The process is repeated and the coil will be charged to a high current. A full rectifier can also be found in [30][31]. Depending on how the switches are operated, the flux pump can be classified into the following categories.

\section{A. Electronic switches based transformer-rectifier flux pumps (ESTRFP)}

In Oomen's work [32], MOSFET arrays are used for switching. The flux pump can operate at frequencies up to $500 \mathrm{~Hz}$ has fast charging speed, high efficiency, and low field fluctuation. The disadvantage is that the MOSFET array has an on-state resistance which introduces a constant loss when the coil is working on quasi-persistent current mode.

\section{B. Heat switches based transformer-rectifier flux pump (HSTRFP)}

This type of flux pumps can be found in Oomen's work [32] and Iwasa's work [33][34][35]. The switches in these flux pumps are made of $\mathrm{NB}_{3} \mathrm{Sn}$ superconductor or YBCO. The switches are opened by heating the superconductor to above its critical temperature. Due to a thermal switch being slow to operate, this type of flux pumps can only work in a low frequency range.

\section{DC field switches based transformer-rectifier flux pump(DCSTRFP)}

This flux pump is theoretically designed in [36]. $\mathrm{MgB}_{2}$ superconductor is used for switches in the design. The switches are operated by applied high DC field to the switch 
superconductor. High $T_{\mathrm{c}}$ superconductors have a very strong field dependence of critical current density, so that critical current of the switch superconductor decreases sharply with external applied field [36]. By carefully coordinating the current capacity of switches, applied DC field, and secondary current, the switches can be operated alternatively to achieve flux pumping.

\section{VARIABLE RESISTANCE RECTIFIER PUMPS}

In these flux pumps [37], switches $S_{1}$ and $S_{2}$ are not present. $S 2$ is replaced by a section of superconductor in which resistance can be induced, either by flux flow or as a dynamic resistance. Fig. 5 shows a typical arrangement.

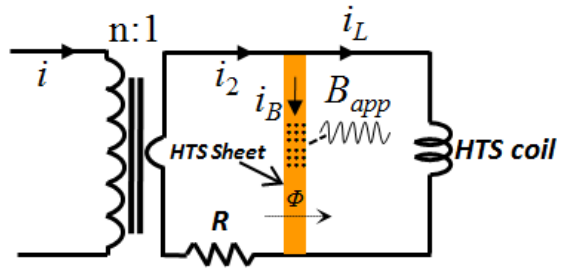

Fig. 5. Variable resistance rectifier pump. [38]

\section{A. Self-Regulating transformer-rectifier flux pump (SRTRFP)}

An asymmetrical alternating current $i_{2}$ is induced in the secondary winding, which has no dc component. The waveform of $i_{2}$ is adjusted, so that its positive peak value exceeds the critical current of the HTS sheet whereas its negative peak value is lower than the critical value (Fig. 6). The current drives the HTS Sheet into the flux flow regime at each positive peak, resulting in a net flux flow across the HTS Sheet. This flux pump saves the trouble of controlling switches, but can only operate in half wave mode.

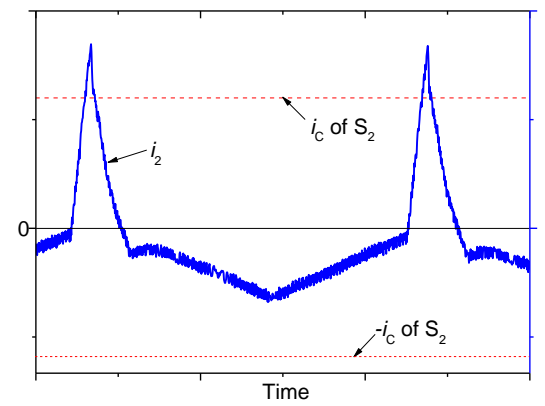

Fig. 6. Current waveform of Self-Regulating transformer-rectifier flux pump [37]

\section{B. AC field switch based transformer-rectifier flux pump (ACSTRFP)}

This type of flux pump was proposed by Geng and Coombs [38]. It is similar to the Self-regulating method but the resistance of the HTS sheet is controlled by ac magnetic field [39]. An alternating secondary current $i_{2}$ with high magnitude and low frequency is induced, and a high frequency ac field is then applied perpendicular to the surface of the HTS sheet when the transport current in the sheet is in one direction. The interaction between the transport current in the sheet and the applied field will induce a net flux flow across the sheet, thus pumping up the load current. The waveforms of the applied field and currents are plotted in Fig. 7(a). If the applied field has a much higher frequency than the current in the sheet and the transformer has a large inductance, we can assume that the current in the sheet is constant during each cycle of the applied field. In this case, a dynamic resistance [40][41][42]model can be used to describe the flux flow process. As shown in Fig. 7(b). At lower frequencies this flux pump could also work as long as the net flux flow across the sheet during each alternating current cycle is non-zero. This flux pump can also operate as a full wave rectifier.

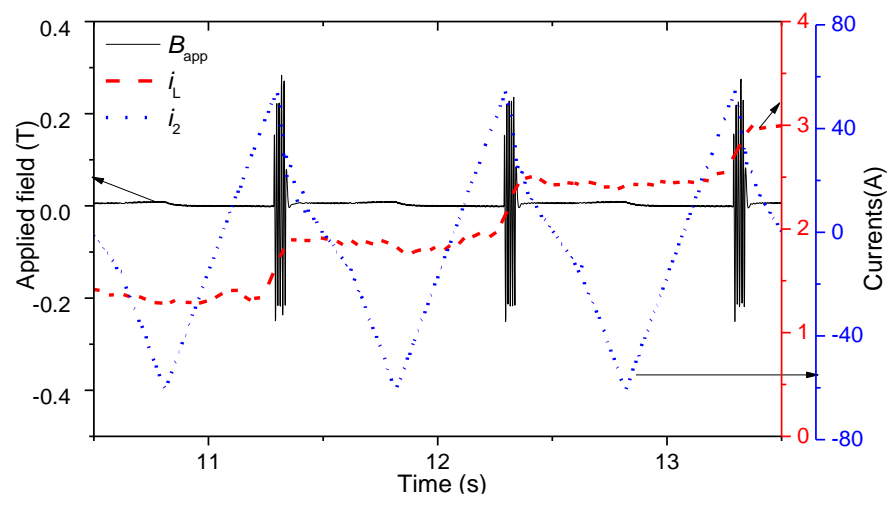

(a) Waveforms of secondary current $i_{2}$, applied field $B_{a p p}, \&$ load current $i_{\mathrm{L}}$.[38]

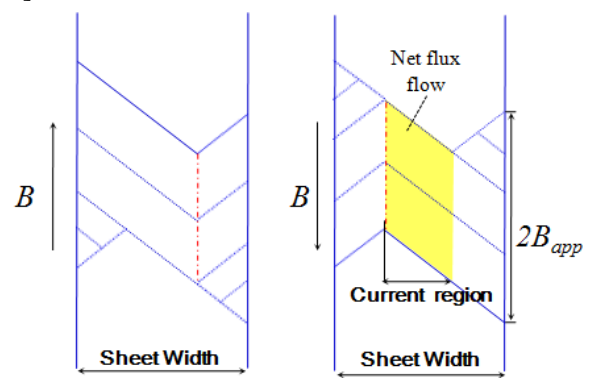

(b) Flux flow mechanism in the HTS sheet.[40][41]

Fig. 7. Transformer-rectifier flux pump based on AC field controlled switch.

\section{General Model For HTS Flux PuMPS}

\section{A. A general circuit model}

A general equivalent circuit [43] for flux pumps is shown in Fig. 8 , this describes both travelling wave flux pumps and transformer-rectifier flux pumps. For travelling wave based flux pumps, the resistances R1 and R2 move with the wave.

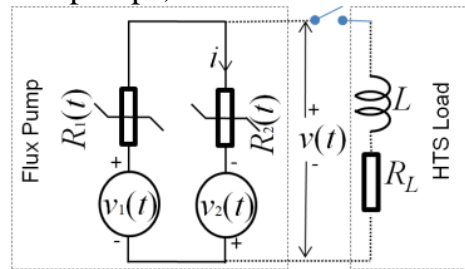

Fig. 8. General flux pump model [43]

The voltage $v_{1}(t)$ or $v_{2}(t)$ reflects the difference between the EMF force induced by external magnetic field and inductive voltage of each side, which represents flux change, i.e.

$$
v_{1}(t)+v_{2}(t)==-d \Phi / d t
$$

Where $\Phi$ is the total flux linking the loop which is periodical. The net average voltage is expressed as [43]: 


$$
V_{A V G}=\frac{1}{T} \int_{0}^{T} v(t) d t=\frac{1}{T} \int_{0}^{T} \frac{-d \Phi / d t}{R_{1}(t)+R_{2}(t)} R_{2}(t) d t
$$

Where $T$ is the period of $\Phi$. The term in the integral is the instantaneous flux motion across $R_{2}(t)$. The integration means that if the net flux flow across $R_{2}(t)$ during the field period is non-zero, a DC voltage will be generated (this would also apply to $R_{1}(t)$ ). It should be noted the resistance $R_{1}(t)$ and $R_{2}(t)$ can either be flux flow resistance of superconductors or ordinary resistance. In this case, ordinary resistance should also be considered as the source of flux leakage.

\section{B. The origins of flux flow}

In transformer-rectifier flux pumps, flux flow is achieved by driving superconductor to flux flow region by high current together with heat and DC field or only by AC field. For travelling wave based flux pumps, the mechanism is similar and is described in detail in[43].

\section{APPLICATIONS OF FLUX PUMPS AND COMPARISON}

\section{A. Applications and progress}

HTS flux pumps can be used to either pump up the current in HTS magnets or maintain a constant current. In [33], a microampere flux pump was designed to compensate the slight current decay in HTS insert of NMR magnets. In [23], Walsh and co-workers exemplified using a rotating permanent magnets based flux pump to charge a 0.4H NMR magnet to $2 \mathrm{~T}$ in 2.5 hours. It is also shown that the field variation is only $18 \mathrm{ppm}$ when the flux pump is used to maintain the field. Researchers in Victoria University of Wellington developed an exciter for HTS rotor windings [45][46][47] They also achieved through-wall operation of flux pump [48], which for the first time moves the flux pump outside the cryogenic system.

\section{B. Comparison of existing flux pumps}

RTWFP is based on a motor, so it is not flexible to control. There may also be noise from the motor. The flux pump has to keep working at a constant speed to maintain a field, otherwise the acceleration or deceleration will cause undesirable fluctuations. This means loss is induced consistently, and a higher load current means a higher loss. This flux pump, however, is suitable for a powering rotor which rotates itself and does not need very stable field, for example in a motor or generator.

LTWFP is more controllable than RTWFP. The drawback is that the behavior is not exactly predictable, so the optimization has to be based on trial and error. Unnecessary loss is also incurred.

ESTRFP is based on power electronic devices which switch fast but have high off-state resistances. It is suitable for high power high frequency applications rather than maintaining a stable field.

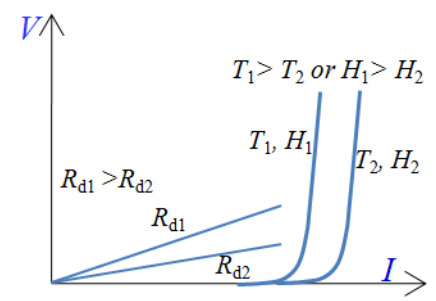

Fig. 9. Operating lines of ACSTRFP (straight lines, $R_{\mathrm{d} 1}$ and $R_{\mathrm{d} 2}$ is the slope of each line), HSTRFP, DCSTRFP, and SRTRFP (exponential lines, which can be shifted by temperature and DC field).

Both HSTRFP and DCSTRFP can operate in full wave mode. DCSTRFP is superior to HSTRFP because a DC field controlled switch operates much faster than a heat controlled one. SRTRFP is the simplest it does not need a switch at all, but it cannot operate a full wave mode. All of them operate at flux flow region of the V-I curve of high $T_{\mathrm{c}}$ superconductor, as shown in Fig. 9. The sharp slope makes it difficult for accurate control. And the switches have to work at high current.

ACSTRFP can be considered as the optimized and simplified version of $L T W F P$. The amount of flux per field cycle can be accurately controlled, thus making it possible to maintain a load field with extremely high stability. As shown in Fig. 9, the switch V-I curve is linear. However, this flux pump is not perfect a high frequency magnetic field is required and for large loads developing a large resistance will require longer lengths of superconductor.

Although the travelling wave and the variable resistance type rectifier pumps do not require either switches or the generation of a normal spot and can be reduced to the same equivalent circuit there are important differences. Generating a travelling wave with coils allows different waveforms to be used at different parts of the charging cycle. Generating a travelling wave with a moving magnet presents the obvious possibility of using the motion of a rotor relative to a stator as the driving mechanism.

The variable resistance rectifier types are the most promising. The resistance is generated independently of the magnetic induction. Unlike the travelling wave types the current $i_{\mathrm{B}}$ is independent of $i_{\mathrm{L}}$ and thus the superconducting losses are minimized.

\section{CONCLUSION}

This paper reviews recent progress in flux pumps for HTS coil magnets. Travelling wave flux pumps and transformer rectifier flux pumps of various kinds are introduced. A general model for these flux pumps is presented and the underlying physics are explained. The applications of these flux pumps are addressed. This paper will be beneficial for researchers and engineers in the understanding of HTS flux pumps

\section{ACKNOWLEDGMENT}

J. Geng would like to acknowledge Cambridge Trust for offering Cambridge International Scholarship to support his study in Cambridge. 


\section{REFERENCES}

[1] M. Sugano et al., "Reversible strain dependence of critical current in 100 A class coated conductors," IEEE Trans. Appl. Supercond., vol. 15, no. 2, pp. 3581-3584, 2005.

[2] H. W. Weijers et al., "High field magnets with HTS conductors," IEEE Trans. Appl. Supercond., vol. 20, no. 3, pp. 576-582, Jun. 2010.

[3] S. Hahn, D. K. Park, J. Voccio, J. Bascuñán, and Y. Iwasa, "No-Insulation (NI) HTS inserts for $>1 \mathrm{GHz}$ LTS/HTS NMR magnets," IEEE Trans. Appl. Supercond., vol. 22, no. 3, Jun. 2012, Art. ID 4302405.

[4] T. Tosaka et al., "Development of a persistent current switch for HTS magnets," IEEE Trans. Appl. Supercond., vol. 14, no. 2, pp. 1218-1221, June 2004

[5] Y. J. Park, M. W. Lee, H. S. Ann, Y. H. Choi, and H. G. Lee, "A superconducting joint for $\mathrm{GdBa} 2 \mathrm{Cu} 3 \mathrm{O} 7-\delta$-coated conductors," $N P G$ Asia Mater., vol. 6, p. e98, 2014.

[6] M. V. Feigel'man, V. B. Geshkenbein, and V. M. Vinoku, "Flux creep and current relaxation in high-Tc superconductors," Phys. Rev. B, vol. 43, no. 4, , pp. 6263-6265, Mar. 1991.

[7] Y. G. Park et al., "Experimental analysis on initial current decay characteristics of persistent-mode HTS coil by external alternating magnetic field," IEEE Trans. Appl. Supercond., vol. 25, no. 3, Jun. 2015, Art. no. 3601204.

[8] J. Geng, H. Zhang, and T. A. Coombs, “Angular Dependence of Persistent Current Decay of YBCO Coil under AC magnetic field", to be submitted to Supercond. Sci. Technol..

[9] T. Coombs"superconducting systems", patent application number WO2007045929, 2007,

[10] Coombs, T. , Hong, Z., Zhu, X, "A thermally actuated superconducting flux pump" ,. Physica C 4683 pp 153-159, 2008

[11] C. Hoffmann, D. Pooke, and A. D. Caplin, "Flux pump for HTS magnets," IEEE Trans. Appl. Supercond., vol. 21, no. 3, pp. 1628-1631,Jun. 2011.

[12] Y. D. Chung, "Design and performance of compensator for decremental persistent current in HTS magnets using linear type magnetic flux pump," Cryogenics, vol. 44, pp. 839-844, 2004.

[13] Y. D. Chung, I. Muta, T. Hoshino, and T. Nakamura, "Characteristics of a persistent current compensator for superconducting NMR magnets using linear type magnetic flux pump," IEEE Trans. Appl. Supercond., vol. 15, no. 2, pp. 1338-1341, June 2005.

[14] Y. D. Chung, T. Hoshino, and T. Nakamura, "Current pumping performance of linear-type magnetic flux pump with use of feedback control circuit system," IEEE Trans. Appl. Supercond., vol. 16, no. 2, pp. $1638-1641,2006$.

[15] T. Nakamura, M. Sugano, T. Doi, and N. Amemiya, "Flux pump effect of HTS films in a traveling magnetic field," IEEE Trans. Appl. Supercond., vol. 20, no. 3, pp. 1033-1036, Jun. 2010.

[16] Z. Bai et al., "A newly developed pulse-type microampere magnetic flux pump," IEEE Trans. Appl. Supercond., vol. 20, no. 3, pp. 1667-1670, Jun. 2010.

[17] Z. Bai, G. Yan, C. Wu, S. Ding, and C. Chen, "A novel high temperature superconducting magnetic flux pump for MRI magnets," Cryogenic, vol. 50 , no. 10, pp. 688-692, Oct. 2010.

[18] Z. Bai, C. Chen, Y. Wu, and Z. Zhen, "Effect of various pulse wave forms for pulse-type magnetic flux pump," Cryogenics, vol. 51, no. 9, pp. 530533, Sep. 2011.

[19] Wang, W. et al Direct measurement of the vortex migration caused by traveling magnetic wave" Applied Physics Letters 104, 3, 0326022014

[20] L. Fu, K. Matsuda, and T. A. Coombs, "Linear Flux Pump Device Applied to HTS Magnets: Further Characteristics on Wave Profile, Number of Poles, and Control of Saturated Current," IEEE Trans. Appl. Supercond., vol. 26, no. 3, Apr., 2006, Art. ID 0500304.

[21] L. Fu, K. Matsuda, M. Baghdadi, and T. Coombs, "Linear flux pump device applied to high temperature superconducting (HTS) magnets," IEEE Trans. Appl. Supercond., vol. 25, no. 3, Jun. 2015, Art. ID 4603804.

[22] L. Fu, K. Matsuda, T. Lecrevisse, Y. Iwasa, and T. Coombs, "Linear flux pump device applied to high temperature superconducting (HTS) magnets," Supercond. Sci. Technol., vol. 29, no. 4, Feb. 2016, Art. no. 04LT01.

[23] R. M. Walsh, R. Slade, P. Donald, and C. Hoffmann, "Characterization of current stability in an HTS NMR system energized by an HTS flux pump," IEEE Trans. Appl. Supercond., vol. 24, no. 3, Jun. 2014, Art. ID 4600805 .
[24] C. Hoffmann, R. Walsh, E. Karrer-Mueller, and D. Pooke, "Design parameters for an HTS flux pump," Phys. Proc., vol. 36, pp. 1324-1329, 2012.

[25] T. A. Coombs, J. F. Fagnard, and K.Matsuda, "Magnetization of 2-G coils and artificial bulks," IEEE Trans. Appl. Supercond., vol. 24, Oct. 2014, Art. ID 8201005.

[26] Z. Jiang, K. Hamilton, N. Amemiya, R. A. Badcock, and C. W. Bumby, "Dynamic resistance of a high- $T_{c}$ superconducting flux pump," Appl. Phys. Lett., vol. 105, no. 11, Sep. 2014, Art. ID 112601.

[27] S. Lee et al., "Persistent Current Mode Operation of A 2G HTS Coil With A Flux Pump," IEEE Trans. Appl. Supercond., vol. 26, no. 4, Jun. 2016, Art. ID 0606104.

[28] Z. Jiang et al., "Impact of flux gap upon dynamic resistance of a rotating HTS flux pump," Supercond. Sci. Technol., vol. 28, no. 11, Nov. 2015, Art. ID 115008.

[29] J. Geng et al., "Voltage-ampere characteristics of YBCO coated conductor under inhomogeneous oscillating magnetic field," Appl. Phys. Lett., vol. 108, no. 26, Jun. 2016, Art. no. 262601.

[30] L. J. M. van de Klundert and H. H. J. ten Kate, "Fully superconducting rectifiers and flux pumps Part 1: Realized methods for pumping flux," Cryogenics, vol. 21, no. 4, pp. 195-206, Apr. 1981.

[31] L. J. M. van de Klundert and H. H. J. ten Kate, "On fully superconducting rectifiers and flux pumps. A review. Part 2: Commutation modes, characteristics and switches," Cryogenics, vol. 21, no. 5, pp. 267-277, May 1981.

[32] M. P. Oomen et al., "HTS flux pump for cryogen free HTS magnets," IEEE Trans. Appl. Supercond., vol. 15, no. 2, pp. 1465-1468, Jun. 2005.

[33] Y. Iwasa, "Microampere flux pumps for superconducting NMR magnets part 1 basic concept and microtesla flux measurement," Cryogenics, vol. 41, pp. 385-391, 2001.

[34] S. K. Jeong, H. Lee, and Y. Iwasa, "Superconducting flux pump for high temperature superconductor insert coils of NMR magnets," Advances in Cryogenic Engineering, vol. 48, pp. 305-312, 2002.

[35] H. Lee, H. M. Kim, and Y. Iwasa, "A flux pump for NMR magnets," IEEE Trans. Appl. Supercond., vol. 13, no. 2, pp. 1640-1643, 2003.

[36] S. Ishmael, C. Goodzeit, P. Masson, R. Meinke, and R. Sullivan, "Flux pump excited double-helix rotor for use in synchronous machines," IEEE Trans. Appl. Supercond., vol. 18, no. 2, pp. 693-696, Jun. 2008.

[37] J. Geng and T. A. Coombs, "An HTS flux pump operated by directly driving a superconductor into flux flow region in the E-J curve," Supercond. Sci. Technol., vol. 29, no. 9, Jul. 2016, Art. no. 095004.

[38] J. Geng and T. A. Coombs, "Mechanism of a high-Tc superconducting flux pump: Using alternating magnetic field to trigger flux flow," Appl. Phys. Lett., vol. 107, no. 14, Oct. 2015, Art. no. 142601.

[39] J. Geng et al., "HTS Persistent Current Switch Controlled by AC Magnetic Field," IEEE Trans. Appl. Supercond., vol. 26, no. 3, Apr., 2006, Art. ID 6603304.

[40] J. Geng et al., "Operational research on a high-Tc rectifier-type superconducting flux pump," Supercond. Sci. Technol., vol. 29, no. 3, Feb. 2016, Art. no. 035015.

[41] G. P. Mikitik and E. H. Brandt, Phys. Rev. B 64, 092502 (2001).

[42] Uksusman A, Wolfus Y, Friedman A, Shaulov A and Yeshurun Y 2009 J. Appl. Phys. 105093921

[43] J. Geng et al., "Origin of dc voltage in type II superconducting flux pumps: field, field rate of change, and current density dependence of resistivity," J. Phys. D: Appl. Phys., vol. 49, no. 11, Feb. 2016, Art. no. 11LT01.

[44] C. W. Bumby, Z. Jiang, A. E. Pantoja, and R. A. Badcock, "Anomalous open-circuit voltage from a high-Tc superconducting dynamo," Appl. Phys. Lett., vol. 108, no. 12, Mar. 2016, Art. ID 122601.

[45] C. W. Bumby et al., "Development of a brushless HTS exciter for a 10 kW HTS synchronous generator," Supercond. Sci. Technol., vol. 29, no. 2, 2016, Art. no. 024008.

[46] Z. Jiang, C. W. Bumby, R. A. Badcock, and H.-J. Sung, "A novel rotating HTS flux pump incorporating a ferromagnetic circuit," IEEE Trans Appl. Supercond., vol. 26, no. 2, Mar. 2016, Art. ID 4900706.

[47] H.-J. Sung et al., "Design and heat load analysis for a 12 MW HTS wind power generator module employing brushless HTS exciter," IEEE Trans. Appl. Supercond., vol. 26, no. 4, Jun. 2016, Art. ID 5205404.

[48] C. W. Bumby et al., "Through-Wall Excitation of a Magnet Coil by an External-Rotor HTS Flux Pump," IEEE Trans. Appl. Supercond., vol. 26, no. 4, Jun. 2016, Art. ID 0500505. 